\title{
Neonatal outcome in maternal genital tract group B streptococcal colonization
}

\author{
Vijayan Sharmila ${ }^{1}$, Thirunavukkarasu Arun Babu ${ }^{1}$, Latha Chaturvedula ${ }^{2}$
}

\author{
${ }^{1}$ Department of Pediatrics, Indira Gandhi Medical College and Research Institute, Puducherry, India \\ ${ }^{2}$ Department of Obstetrics and Gynaecology, JIPMER, Puducherry, India
}

Received: 25 July 2016

Accepted: 26 August 2016

\section{*Correspondence: \\ Dr. Thirunavukkarasu Arun Babu, \\ E-mail: babuarun@yahoo.com}

Copyright: $\odot$ the author(s), publisher and licensee Medip Academy. This is an open-access article distributed under the terms of the Creative Commons Attribution Non-Commercial License, which permits unrestricted non-commercial use, distribution, and reproduction in any medium, provided the original work is properly cited.

\begin{abstract}
Background: Group B streptococcus (GBS) is a leading cause of early onset neonatal infections and associated morbidity and mortality in the western world. The objective of this study was to assess neonatal outcome in maternal genital tract Group B streptococcal (GBS) colonization.

Methods: Pregnant women with gestational age greater than 24 weeks were screened for vaginal GBS colonization during their regular visits to antenatal clinic. The vaginal swabs were inoculated in Todd Hewitt broth and later subcultured on blood agar for isolation of GBS. The patients were followed up till delivery. Neonatal characteristics like symptoms of clinical sepsis, screening tests for sepsis, culture positivity, low birth weight, preterm delivery and survival at 1 month were recorded and analyzed.

Results: A total of 316 pregnant women were enrolled in the study. GBS was isolated from 7 patients (2.3\%). A statistically significant increase in occurrence of PROM in women with GBS colonization was noted. However, none of the babies born to women with GBS colonization developed any clinical or proven sepsis, low birth weight or any other negative outcomes.

Conclusions: GBS colonization rate was extremely low in our study. There was significant association between maternal genital tract colonization of GBS and occurrence of PROM. However, no neonatal adverse effects were found to be associated with GBS colonization in pregnancy.
\end{abstract}

Keywords: Group B streptococci, Genital tract, Colonization, Neonatal outcome, Premature rupture of membranes, Neonatal sepsis

\section{INTRODUCTION}

Group B streptococcus (GBS) is a leading cause of early onset neonatal infections and associated morbidity and mortality in the western world. However in India, the spectrum of group B streptococcal disease remains a largely under-recognized problem. ${ }^{1}$ It is believed that GBS is not a significant pathogen causing neonatal sepsis when it comes to the developing world. Universal screening for maternal group B streptococcus (GBS) infection and intra-partum antibiotic prophylaxis of all colonized women is currently the recommended standard of care in North America. ${ }^{1-3}$ In India, routine GBS screening during pregnancy is not done and instead intrapartum antibiotic prophylaxis is given in cases with premature rupture of membranes (PROM).

In Neonatal GBS disease, two distinct clinical syndromes are recognized, early-and late-onset disease. Early onset GBS disease presents within the first 7 days of life. The transmission of early-onset disease is often vertical. ${ }^{4}$ Infection may be acquired by the intra amniotic route, or directly during passage through the birth canal. Lateonset disease develops in infants after 7 days to 3 months of age. Transmission can be either horizontal or vertical. Due to the low incidence of GBS colonisation during 
pregnancy in India, there is limited data about the neonatal outcome in maternal GBS genital tract colonization. ${ }^{1,5}$ We performed this study to find out the association between maternal genital colonization with GBS during pregnancy and neonatal outcome.

\section{METHODS}

A prospective observational cohort study was done in a tertiary care teaching hospital located in South India. This study was done over a period of 20 months from September 2006 to June 2008. All pregnant women with gestational age greater than 24 weeks, attending the antenatal out-patient department during the study period were enrolled in the study and followed up till delivery. Those with history of intake of antibiotics during the past two weeks and/or preexisting medical disorders complicating pregnancy such as diabetes mellitus, hypertension, tuberculosis, heart disease, epilepsy, thyroid dysfunction and jaundice were excluded from the study. Pregnant women with known risk factors for PROM and preterm labor such as cervical incompetence, amniocentesis, cervical surgery during pregnancy, multiple gestations, prior PROM, prior preterm delivery and bleeding in the first trimester were also excluded. The subjects were briefed about the study and a written informed consent was obtained in the vernacular language. This study was approved by the institute's research and ethical committees.

A brief clinical history was obtained and clinical examination was performed. Parameters such as age, parity, period of gestation and other relevant data were noted. The pregnant women enrolled in the study were screened for vaginal colonization with GBS during their regular visits to the antenatal clinic. Low vaginal swabs were obtained prior to speculum examination using two sterile cotton swabs inserted just beyond hymenal ring. The vaginal swabs were immediately placed in separate Todd-Hewitt broths, an enrichment medium for GBS and sent to the Microbiology laboratory. The Todd-Hewitt broth was initially incubated for 24 hours at $37{ }^{\circ} \mathrm{C}$ in a $5 \% \mathrm{CO}_{2}$ atmosphere, followed by subculture onto $5 \%$ sheep blood agar, which was further incubated for $18-24$ hours at $37^{\circ} \mathrm{C}$ in a candle jar.

The blood agar plates were examined at 24 hours and 48 hours for the presence of GBS. GBS was identified based on the colony morphology, beta hemolysis, characteristic biochemical reactions (catalase negative, sodium hippurate hydrolysis positive, CAMP test positive, bacitracin resistance) and was confirmed by latex agglutination test.

The pregnant women were followed up till delivery and the characteristics of their babies were noted. Presence of clinical symptoms suggestive of sepsis, screening tests for sepsis, culture positivity, low birth weight, preterm delivery and survival at 1 month were recorded and analyzed.

Data entry and analysis were done using statistical software SPSS for Windows Version 16.0 (SPSS Inc, Chicago, IL, USA). Percentages were calculated for categorical variables. Means and standard deviations (SD) were calculated as required for numerical variables. The Chi-square test and Fisher's exact test were used. The relative risk, $95 \%$ confidence interval and $p$ values of outcome parameters were calculated.

\section{RESULTS}

A total of 316 pregnant women were included in the study. The age of the participants ranged from 18 to 39 years with a mean \pm SD of $23.28 \pm 2.95$. GBS was isolated from 7 out of 300 patients, corresponding to a colonization rate of $2.3 \%$. The mean age of the women with GBS colonization was $24.57 \pm 2.23$ and the mean age of pregnant women without GBS colonization was 23.25 \pm 2.96 . Out of the 300 pregnant women, $195(65 \%)$ were primi gravida, while $105(35 \%)$ were multigravida.

Table 1: Univariate analysis to assess the association between GBS colonization and neonatal outcome.

\begin{tabular}{|lllll|}
\hline Outcome parameters & $\begin{array}{l}\text { GBS colonisation } \\
(\mathrm{n}=7)\end{array}$ & $\begin{array}{l}\text { No GBS colonisation } \\
(\mathrm{n}=293)\end{array}$ & RR $(95 \% \mathrm{CI})$ & P value \\
\hline Suspected clinical sepsis & 2 & 38 & $2.2(0.65-7.37$ & 0.200 \\
\hline Positive septic screen & 1 & 17 & $2.46(0.37-16.01)$ & 0.343 \\
\hline GBS culture positive sepsis & 0 & 0 & - & - \\
\hline Non GBS culture positive sepsis & 0 & 3 & $5.3(0.29-94.36)$ & 0.256 \\
\hline Survival at 1 month & 7 & 291 & $7.3(0.38-141.0)$ & 0.185 \\
\hline Low birth weight & 3 & 87 & $1.4(0.60-3.45)$ & 0.4102 \\
\hline Preterm delivery & 0 & 8 & - & 1.000 \\
\hline PROM & 3 & 14 & $12.4(3.03-251.38)$ & 0.0046 \\
\hline
\end{tabular}


The results of the univariate analysis performed to assess the association between GBS colonization and adverse neonatal outcome is detailed in Table 1 . There was no statistically significant increase in occurrence of bad neonatal outcomes in neonates born to pregnant women with GBS colonization.

\section{DISCUSSION}

The effect of colonization of the maternal genito-urinary tract with GBS on the obstetric outcome is unclear and whether maternal GBS colonization is a predictor of adverse obstetric outcome remains controversial. ${ }^{6-8}$

Although the maternal colonization with GBS has been widely accepted as an important cause of early-onset neonatal sepsis, the effect of GBS colonization on the outcome of the pregnancy remains controversial. In this prospective study, we observed a significantly increased occurrence of PROM in women with GBS colonization. PROM is defined as rupture of membranes before onset of uterine contractions. It occurs in $3 \%$ of all pregnancies at or near term. ${ }^{9}$ Increased incidence of PROM was observed among women colonized with GBS in few studies. ${ }^{6,8}$ Some studies also found that there was no significant association between maternal GBS colonization and PROM. ${ }^{10,11}$ Therefore, the role of GBS colonization in causation of PROM is uncertain. ${ }^{4,6,8}$

Preterm labour is the other common adverse obstetric outcome suggested to be associated with GBS colonization. ${ }^{6,7,12}$ Some studies have shown that pregnant women who are vaginal carriers of GBS have an increased risk of preterm labour, while other studies do not support such an association between prematurity and GBS colonization. ${ }^{1,3,6,13}$ In our study, we did not find a significant association between GBS colonization and prematurity. Our failure to prove a significant association between these two adverse obstetric outcomes and GBS colonization could be partially due to the small number of GBS positive cases, which is a limitation of this study. However, a systemic review has also concluded that there is no significant association between maternal GBS colonization during pregnancy and preterm delivery. ${ }^{14}$

There was no significant increase in the number of suspected or proven cases of neonatal sepsis in the GBS colonization group. There were 2 cases of suspected clinical sepsis in GBS colonization group which required treatment with antibiotics for 48 hours but none of these cases had a positive blood culture. There was no risk of preterm delivery or low birth weight noted in the GBS colonized group in comparison to the mother without GBS colonization. All 7 babies survived the first month. Thus GBS colonization does not appear to significantly increase the risk of sepsis in neonates.

\section{CONCLUSION}

The incidence of GBS colonization was very low in our study. GBS colonization was associated with a significantly increased risk of PROM. However, there was no significant association between GBS colonization and prematurity, neonatal sepsis or survival at 1 month.

Funding: No funding sources

Conflict of interest: None declared

Ethical approval: The study was approved by the Institutional Ethics Committee

\section{REFERENCES}

1. Shet A, Ferrieri P. Neonatal and maternal group B streptococcal infections: a comprehensive review. Indian J Med Res. 2004;120:141-50.

2. Regan JA, Klebanoff MA, Nugent RP. The epidemiology of group B streptococcal colonization in pregnancy. Vaginal infections and prematurity study group. Obstet Gynecol. 1991;77:604-10.

3. Namavar JB, Poorarian S, Poorbarfehee S. The prevalence and adverse effects of group B streptococcal colonization during pregnancy. Arch Iran Med. 2008;11:654-7.

4. Boyer KM, Gadzala CA, Kelly PD, Burd LI, Gotoff SP. Selective intra-partum chemoprophylaxis of neonatal group B streptococcal early-onset disease. II. Predictive value of prenatal cultures. J Infect Dis. 1983;148:802-9.

5. Dechen TC, Sumit K, Ranabir P. Correlates of Vaginal colonization with group B streptococci among pregnant women. J Glob Infect Dis. 2010;2(3):236-41.

6. Regan JA, Chao S, James LS. Premature rupture of membranes, preterm delivery, and group B streptococcal colonization of mothers. Am J Obstet Gynecol. 1981;141:184-6.

7. McDonald H, Vigneswaran R, O'Loughlin JA. Group B streptococcal colonization and preterm labour. Aust N Z J Obstet Gynaecol.1989;29:291-3.

8. Feikin DR, Thorsen P, Zywicki S, Arpi M, Westergaard JG, Schuchat A. Association between colonization with group B streptococci during pregnancy and preterm delivery among Danish women. Am J Obstet Gynecol. 2001;184:427-33.

9. Kaur BA, Vats U, Nandanwar YS. Role of serial ultrasound assessment in prom patients and its outcome (prospective study). Bombay Hospital Journal. 2009;51:163-6.

10. Kubota T. Relationship between maternal group B streptococcal colonization and pregnancy outcome. Obstet Gynecol. 1998;92:926-30.

11. Garland SM, Kelly N, Ugoni AM. Is antenatal group B streptococcal carriage a predictor of adverse obstetric outcome? Infect Dis Obstet Gynecol. 2000;8:138-42.

12. Glass NE, Schulkin J, Chamany S, Riley LE, Schuchat A, Schrag S. Opportunities to reduce 
overuse of antibiotics for perinatal group B streptococcal disease prevention and management of preterm premature rupture of membranes. Infect Dis Obstet Gynecol. 2005;13:5-10.

13. Romero R, Mazor M, Oyarzun E, Sirtori M, Wu YK, Hobbins JC. Is there an association between colonization with group B Streptococcus and prematurity? J Reprod Med. 1989;34:797-801.

14. Valkenburg-van den Berg AW, Sprij AJ, Dekker FW, Dorr PJ, Kanhai HH. Association between colonization with group B streptococcus and preterm delivery: a systematic review. Acta Obstet Gynecol Scand. 2009;88:958-67.

Cite this article as: Sharmila V, Babu TA,

Chaturvedula L. Neonatal outcome in maternal genital tract group B streptococcal colonization. Int J Reprod Contracept Obstet Gynecol 2016;5:3444-7. 